\title{
Ant colony optimization technique in optimal capacitor placement and sizing problem in unbalanced electrical distribution system
}

\begin{abstract}
The optimal capacitor placement problem involves determination on the type, number, location and size of capacitors to be placed in a distribution system for the attainment of energy efficiency. The main objectives of the capacitors placement and sizing are to reduce total line loss and energy consumption while satisfying the operational constraints. This paper presents the optimal capacitor placement and sizing problems solved by using the Ant Colony Optimization (ACO) technique with the integrated of circuitry unbalanced electrical distribution modeled in SIMULINK $®$ MATLAB $®$ software. The proposed technique was tested on a modified IEEE 13-bus unbalanced radial distribution system and the results revealed that the proposed technique has the merit in achieving optimal solution for addressing the problems.
\end{abstract}

Keyword: Ant colony optimization; Optimal capacitors placement; Power loss index; Sizing; Unbalanced electrical distribution system 